\title{
Efeito do ataque de Alabama argillacea no crescimento vegetativo e sua relação com a fenologia do algodoeiro(1)
}

\begin{abstract}
Ednaldo da Silva Quirino(2) e José Janduí Soares ${ }^{(2)}$
Resumo - O objetivo deste trabalho foi estudar o efeito do ataque do curuquerê (Alabama argillacea Hübner, 1818), no desenvolvimento vegetativo do algodoeiro e sua relação com a fenologia da planta. Foram utilizadas as cultivares CNPA 7H e CNPA Precoce 2, e semeadas em vasos de plástico com capacidade para $10 \mathrm{~kg}$ de solo, mantendo-se uma planta por vaso após o desbaste. O delineamento experimental utilizado foi inteiramente casualizado, com sete tratamentos e quatro repetições. Foram utilizadas lagartas de terceiro ínstar de $A$. argillacea, provenientes de criação massal mantida em laboratório. A infestação por estas lagartas teve início 40 dias após o plantio, mediante a identificação das folhas que caracterizavam os tratamentos. Foram avaliadas as variáveis diâmetro caulinar e altura de plantas, em 1996; e em 1997, foi acrescentada a variável área foliar. O ataque de A. argillacea afeta o diâmetro caulinar e a altura das plantas em ambas as cultivares e em qualquer fase de desenvolvimento do algodoeiro. Com relação à área foliar, os maiores decréscimos foram verificados nos tratamentos que tiveram as folhas dos ramos principais consumidas; o tratamento mais afetado foi aquele em que o ataque ocorreu após a floração.
\end{abstract}

Termos para indexação: Gossypium hirsutum, área foliar, caule, etapas de desenvolvimento da planta, pragas das plantas.

\section{Effect of Alabama argillacea attack on vegetative growth and its relationship with cotton phenology}

Abstract - This work was carried out to study the effect of cotton leaf worm attack on vegetative growth and its relation with plant phenology. The CNPA 7H and CNPA Precoce 2 cultivars were planted in plastic pots with capacity for $10 \mathrm{~kg}$ of soil, and one plant per pot was maintained after pruning. A completely randomized block design was used with seven treatments and four replications. Thirdinstar caterpillars of Alabama argillacea were used in the experiment, which were originated from a massal rearing creation kept in laboratory. Infestation with caterpillars started 40 days after the planting by identification of leaves that characterized the treatments. The variables analyzed were plant diameter and height in 1996, and plant diameter and height and leaf area in 1997. The attack on primary branch leaves caused reductions in plant diameter and height for both cultivars, whichever growth stage of the plant. In regard to leaf area, greater decreases were observed for treatments that had the main branch leaves wasted away. The most affected one was that in which the attack has occurred after flowering.

Index terms: Gossypium hirsitum, leaf area, stems, plant developmental stages, pests of plants.

\section{Introdução}

O algodoeiro herbáceo (Gossypium hirsutum L. raça latifolium Hutch) é atacado por diversas pragas que causam prejuízos à cultura, dentre as quais se des-

(1) Aceito para publicação em 17 de outubro de 2000 .

(2) Embrapa-Centro Nacional de Pesquisa de Algodão, Caixa Postal 174, CEP 58107-720 Campina Grande, PB E-mail: esquirino@zipmail.com.br, soares@cnpa.embrapa.br taca o curuquerê (Alabama argillacea Hüebner, 1818), considerada a segunda praga mais danosa à lavoura algodoeira (Soares et al., 1997a). No Nordeste, é considerada praga-chave ou primária, e pode surgir logo após a emergência das plantas. Seu ataque destrói a área foliar, provocando queda acentuada na produção (Cavalcante \& Cavalcante, 1981). Segundo Cavalcanti (1977) e Bleicher et al. (1983), as plantas não são resistentes a grandes perdas de área foliar nos primeiros 45 dias de desenvolvimento. Outros 
autores (Marchini, 1976; Falcon \& Dayl, 1977; Nakano et al., 1981; Silva et al., 1981) avaliaram o efeito de vários níveis de desfolha pelo curuquerê na produção do algodoeiro. De acordo com Marchini (1976), o curuquerê consome durante seu estádio de desenvolvimento $66 \mathrm{~cm}^{2}$ de uma folha de algodão. Para Alvarez \& Sanchez (1982), o consumo observado em todos os ínstares de Alabama argillacea é de $88 \mathrm{~cm}^{2}$, enquanto Johnsen (1984) verificou que à temperatura constante de $27,5^{\circ} \mathrm{C}$, o consumo médio de uma lagarta foi de $117,95 \mathrm{~cm}^{2}$, e que o último ínstar consumiu aproximadamente $73 \%$ do total.

Beltrão \& Azevedo (1993) observaram perdas significativas no desenvolvimento da planta após a retirada das folhas da haste principal. Soares et al. (1997b) verificaram que a remoção das folhas da haste principal do algodoeiro reduz drasticamente a produção, o número de ramos frutíferos, e a altura de plantas, entre outras variáveis.

As informações sobre a participação das folhas da haste principal e das folhas dos ramos frutíferos para a definição da produção do algodoeiro são numerosas, considerando-se a remoção manual dessas folhas; contudo, pouca ou nenhuma informação existe, considerando-se os danos provocados diretamente por insetos nas folhas (Jácome et al., 2001).

Objetivou-se, com este trabalho, verificar os efeitos do ataque de $A$. argillacea no crescimento vegetativo do algodoeiro e sua relação com a fenologia desta planta.

\section{Material e Métodos}

Os experimentos foram conduzidos na base física da Embrapa-Centro Nacional de Pesquisa de Algodão (CNPA), no Município de Campina Grande, PB, nos anos agrícolas de 1996 e 1997. Foram utilizadas sementes das cultivares de algodoeiro herbáceo CNPA 7H e CNPA Precoce 2 , semeados em vasos de plástico com capacidade para $10 \mathrm{~kg}$ de solo, corrigido em face da análise química nele efetuada, permitindo obter os seguintes resultados: pH 7,0; em mg/kg de solo: $\mathrm{Ca}^{2+}, 17,0 ; \mathrm{Mg}^{2+}, 8,0 ; \mathrm{Na}^{+}$, 1,$10 ; \mathrm{Al}^{3+}, 0,00 ; \mathrm{P}, 216,90$ e matéria orgânica, 6,43\%. Os experimentos foram conduzidos em casa de vegetação, e o delineamento experimental empregado foi o inteiramente casualizado, com sete tratamentos e quatro repeti- ções. Os tratamentos foram os seguintes: 1) consumo das folhas do ramo principal durante o ciclo da cultura; 2 ) consumo das folhas dos ramos frutíferos durante o ciclo da cultura; 3) consumo das folhas do ramo principal até o aparecimento das flores; 4) consumo das folhas dos ramos frutíferos até o aparecimento das flores; 5) consumo das folhas do ramo principal a partir do aparecimento das flores; 6) consumo das folhas dos ramos frutíferos a partir do aparecimento das flores; 7) testemunha.

Foram utilizadas, no experimento, lagartas de terceiro ínstar de $A$. argillacea, provenientes da criação massal mantida no Laboratório de Entomologia.

A infestação por lagartas iniciou-se 40 dias após a emergência, mediante a identificação das folhas que caracterizaram os tratamentos. Estas lagartas eram protegidas com uma bolsa de tecido tipo voil.

Foram observadas as seguintes variáveis: diâmetro caulinar de plantas $(\mathrm{mm})$ e altura de plantas $(\mathrm{cm})$ aos 40 , 60 e 80 dias após a emergência (DAE). Além destas variáveis, mediu-se também a área foliar $\left(\mathrm{cm}^{2}\right)$, utilizando a fórmula $\log \mathrm{y}=0,045+1,910 \log \mathrm{x}$, onde $\mathrm{x}$ é o comprimento da folha e y é a área foliar.

\section{Resultados e Discussão}

Em relação à cultivar CNPA 7H, no ano de 1996, a variável diâmetro caulinar não apresentou diferença significativa entre os tratamentos, aos 40 e 60 dias após a emergência (DAE) (Tabela 1). No entanto, aos 80 DAE o tratamento 2 diferiu significativamente apenas do tratamento 3 . Não houve diferença significativa entre a testemunha e os tratamentos 3, 4, 5, 6 e 7 .

Quanto à altura das plantas não houve diferença entre os tratamentos nas duas primeiras avaliações (aos 40 e 60 DAE); porém aos 80 DAE o tratamento 2 diferiu significativamente da testemunha e do tratamento 3; e não diferiu significativamente dos tratamentos 4, 5, 6 e 7 (Tabela 1).

Os resultados dos levantamentos efetuados aos 40 e 60 DAE divergem dos encontrados por Jácome (1996), no mesmo período, ao realizar desfolha manual, talvez pelo fato de o inseto atacar de forma gradativa, proporcionando à planta alguma reação. Na desfolha manual, o seccionamento da folha ocorre instantaneamente, pois não há chances de a planta reagir, alterando o diâmetro do caule e a altura. 
Quanto aos levantamentos aos 80 DAE em relação ao diâmetro e à altura, os dados corroboram os já encontrados por Oosterhuis \& Urwiler (1988), Beltrão \& Azevedo (1993) e Jácome (1996), quando realizaram desfolha manual.

Não houve diferença significativa entre os tratamentos em relação ao diâmetro caulinar da cultivar CNPA Precoce 2 aos 40 DAE (Tabela 1). O tratamento 6 diferiu significativamente apenas dos tratamentos 1, 3 e 5 aos 60 DAE, e dos tratamentos 1 e 3 aos 80 DAE, e não diferiu significativamente dos tratamentos 2,4 e 7 aos 60 e 80 DAE.

Com relação à variável altura de plantas, aos 40 DAE, os tratamentos 2, 3 e 6 diferiram significativamente da testemunha (Tabela 1). Aos 60 DAE, os tratamentos 2 e 5 diferiram significativamente do tratamento 1 , e aos $80 \mathrm{DAE}$, os tratamentos 5 e 6 diferiram significativamente do tratamento 1 .

Estes resultados são semelhcantes aos obtidos por Beltrão et al. (1992, 1993) e Soares \& Busoli (1996), que constataram haver maior influência das folhas da haste principal do que das folhas dos ramos frutíferos no desenvolvimento do caule. Beltrão \& Azevedo (1993) constataram haver um decréscimo significativo no desenvolvimento da planta quando retiraram as folhas do ramo principal.
Com relação à cultivar CNPA $7 \mathrm{H}, \mathrm{o}$ tratamento 7 diferiu significativamente dos tratamentos 1, 2, 3, 4 e 5 quanto ao diâmetro caulinar aos 40 DAE (Tabela 2). Aos 60 DAE o tratamento 6 diferiu significativamente apenas dos tratamentos 3 e 5 , e aos 80 DAE, os tratamentos não apresentaram diferenças significativas entre si.

Em relação à altura de plantas, os tratamentos 2 e 7 diferiram significativamente apenas dos tratamentos 3 e 5 aos 40 DAE, e aos 60 DAE, os tratamentos 6 e 7 diferiram significativamente dos tratamentos 1 e 3 (Tabela 2). Aos 80 DAE, os tratamentos 2, 6 e 7 foram os únicos que diferiram significativamente da testemunha. Os tratamentos 2, 4, 5, 6 e 7 não diferiram significativamente entre si.

Tanto em relação ao diâmetro caulinar como em relação à altura de plantas, não houve diferença significativa entre os tratamentos aos 40, 60 e 80 DAE (Tabela 2).

De acordo com estes resultados, a cultivar CNPA Precoce 2 sofreu menos que a cultivar CNPA 7H, por apresentar maior precocidade, característica conhecida como escape ou assincronia fenológica (Lara, 1991). Embora esta característica seja mais estudada com relação ao bicudo-do-algodoeiro, como evidenciaram Nanken et al. (1975) e Beltrão \&

Tabela 1. Resultados médios relativos a diâmetro caulinar $(\mathrm{mm})$ e altura de plantas ( $\mathrm{cm})$, em plantas de algodão cultivares CNPA 7H e CNPA Precoce 2 aos 40, 60 e 80 dias após a emergência (DAE), em 1996. Campina Grande, $\mathrm{PB}^{(1)}$.

\begin{tabular}{|c|c|c|c|c|c|c|}
\hline \multirow[t]{2}{*}{ Tratamento } & \multicolumn{3}{|c|}{ Diâmetro } & \multicolumn{3}{|c|}{ Altura } \\
\hline & $40 \mathrm{DAE}$ & $60 \mathrm{DAE}$ & $80 \mathrm{DAE}$ & $40 \mathrm{DAE}$ & $60 \mathrm{DAE}$ & $80 \mathrm{DAE}$ \\
\hline & \multicolumn{6}{|c|}{ CNPA 7H } \\
\hline 1 & $4,6 \mathrm{a}$ & $5,5 \mathrm{a}$ & $5,6 a b$ & $40,0 \mathrm{a}$ & $48,0 \mathrm{a}$ & $49,3 b$ \\
\hline 2 & $4,8 \mathrm{a}$ & $5,4 \mathrm{a}$ & $6,4 \mathrm{a}$ & $43,0 \mathrm{a}$ & $60,2 \mathrm{a}$ & $66,7 \mathrm{a}$ \\
\hline 3 & $4,5 \mathrm{a}$ & $4,9 a$ & $5,1 b$ & $39,5 \mathrm{a}$ & $47,0 \mathrm{a}$ & $48,0 \mathrm{~b}$ \\
\hline 4 & $4,5 \mathrm{a}$ & $5,2 \mathrm{a}$ & $6, \mathrm{ab}$ & $40,8 \mathrm{a}$ & $54,0 \mathrm{a}$ & $57,2 \mathrm{ab}$ \\
\hline 5 & $4,4 \mathrm{a}$ & $5,6 a$ & $5,5 \mathrm{ab}$ & $37,0 \mathrm{a}$ & $51,7 \mathrm{a}$ & $53,1 \mathrm{ab}$ \\
\hline 6 & $4,7 \mathrm{a}$ & $6,0 \mathrm{a}$ & $5,9 \mathrm{ab}$ & $43,6 a$ & $57,5 \mathrm{a}$ & $60,5 \mathrm{ab}$ \\
\hline 7 & $4,3 \mathrm{a}$ & $5,6 \mathrm{a}$ & $5,6 \mathrm{ab}$ & $38,5 \mathrm{a}$ & $53,2 \mathrm{a}$ & $54,1 \mathrm{ab}$ \\
\hline \multirow[t]{2}{*}{$\mathrm{CV}(\%)$} & 8,56 & 9,66 & 8,46 & 13,22 & 12,67 & 11,96 \\
\hline & \multicolumn{6}{|c|}{ CNPA Precoce 2} \\
\hline 1 & $4,1 \mathrm{a}$ & $4,7 b$ & $4,9 \mathrm{~b}$ & $32,0 \mathrm{ab}$ & $36,0 \mathrm{~b}$ & $37,8 b$ \\
\hline 2 & $4,4 a$ & $5,4 \mathrm{ab}$ & $5,4 \mathrm{ab}$ & $34,7 \mathrm{a}$ & $45,5 \mathrm{a}$ & $46,2 \mathrm{ab}$ \\
\hline 3 & $4,5 \mathrm{a}$ & $5,0 \mathrm{~b}$ & $5,1 \mathrm{~b}$ & $36,5 \mathrm{a}$ & $41,5 \mathrm{ab}$ & $42,8 \mathrm{ab}$ \\
\hline 4 & $4,0 \mathrm{a}$ & $5,3 \mathrm{ab}$ & $5,5 \mathrm{ab}$ & $31,7 \mathrm{ab}$ & $42,5 \mathrm{ab}$ & $43,7 \mathrm{ab}$ \\
\hline 5 & $4,4 a$ & $5,5 b$ & $5,7 \mathrm{ab}$ & $34,0 \mathrm{ab}$ & $45,8 \mathrm{a}$ & $47,0 \mathrm{a}$ \\
\hline 6 & $4,3 \mathrm{a}$ & $5,9 \mathrm{a}$ & $6,1 \mathrm{a}$ & $34,8 \mathrm{a}$ & $45,0 \mathrm{ab}$ & $47,5 \mathrm{a}$ \\
\hline 7 & $4,1 \mathrm{a}$ & $5,2 \mathrm{ab}$ & $5,5 \mathrm{ab}$ & $27,6 \mathrm{~b}$ & $37,7 \mathrm{ab}$ & $39,1 \mathrm{ab}$ \\
\hline $\mathrm{CV}(\%)$ & 6,54 & 3,85 & 6,44 & 7,77 & 9,49 & 9,24 \\
\hline
\end{tabular}

${ }^{(1)}$ Médias seguidas da mesma letra não diferem entre si a $5 \%$ de probabilidade, pelo teste de Tukey. 
Tabela 2. Resultados médios referentes a diâmetro caulinar $(\mathrm{mm})$ e altura de plantas $(\mathrm{cm})$, em plantas de algodão cultivares CNPA 7H e CNPA Precoce 2 aos 40, 60 e 80 dias após a emergência (DAE), em 1997. Campina Grande, $\mathrm{PB}^{(1)}$.

\begin{tabular}{|c|c|c|c|c|c|c|}
\hline \multirow[t]{2}{*}{ Tratamento } & \multicolumn{3}{|c|}{ Diâmetro } & \multicolumn{3}{|c|}{ Altura } \\
\hline & $40 \mathrm{DAE}$ & $60 \mathrm{DAE}$ & $80 \mathrm{DAE}$ & $40 \mathrm{DAE}$ & $60 \mathrm{DAE}$ & $80 \mathrm{DAE}$ \\
\hline & \multicolumn{6}{|c|}{ CNPA 7H } \\
\hline 1 & $4,6 b$ & $6,0 \mathrm{ab}$ & $6,2 \mathrm{a}$ & $30,0 \mathrm{abc}$ & $42,4 \mathrm{bc}$ & $44,6 b$ \\
\hline 2 & $4,7 b$ & $6,5 \mathrm{ab}$ & $7,1 \mathrm{a}$ & $34,6 \mathrm{a}$ & $55,9 \mathrm{ab}$ & $61,5 \mathrm{a}$ \\
\hline 3 & $4,1 b$ & $5,3 b$ & $5,6 \mathrm{a}$ & $25,7 d$ & $38,2 \mathrm{c}$ & $43,2 b$ \\
\hline 4 & $4,7 b$ & $6,1 \mathrm{ab}$ & $6,7 \mathrm{a}$ & 31,2abcd & $48,9 \mathrm{abc}$ & $58,4 \mathrm{ab}$ \\
\hline 5 & $4,2 \mathrm{~b}$ & $6,0 \mathrm{~b}$ & $6,4 \mathrm{a}$ & $27,2 \mathrm{~cd}$ & $44,0 \mathrm{abc}$ & $49,2 \mathrm{ab}$ \\
\hline 6 & $4,8 \mathrm{ab}$ & $6,7 \mathrm{a}$ & $6,9 \mathrm{a}$ & $34,1 \mathrm{abc}$ & $56,2 \mathrm{a}$ & $63,4 \mathrm{a}$ \\
\hline 7 & $5,7 \mathrm{a}$ & $7,4 \mathrm{ab}$ & $7,6 \mathrm{a}$ & $37,7 \mathrm{a}$ & $57,7 \mathrm{a}$ & $64,6 \mathrm{a}$ \\
\hline \multirow[t]{2}{*}{$\mathrm{CV}(\%)$} & 9,95 & 8,28 & 10,42 & 9,60 & 12,12 & 13,66 \\
\hline & \multicolumn{6}{|c|}{ CNPA Precoce 2} \\
\hline 1 & $5,0 \mathrm{a}$ & $6,4 a$ & $7,2 \mathrm{a}$ & $32,1 \mathrm{a}$ & $45,3 \mathrm{a}$ & $48,8 \mathrm{a}$ \\
\hline 2 & $4,7 \mathrm{a}$ & $6,3 a$ & $6,8 \mathrm{a}$ & $29,5 \mathrm{a}$ & $50,6 a$ & $54,3 \mathrm{a}$ \\
\hline 3 & $4,5 \mathrm{a}$ & $6,2 \mathrm{a}$ & $6,5 \mathrm{a}$ & $29,5 a$ & $39,8 \mathrm{a}$ & $43,0 \mathrm{a}$ \\
\hline 4 & $4,7 \mathrm{a}$ & $6,7 \mathrm{a}$ & $7,4 \mathrm{a}$ & $32,1 \mathrm{a}$ & $55,7 \mathrm{a}$ & $65,5 \mathrm{a}$ \\
\hline 5 & $5,7 \mathrm{a}$ & $6,5 \mathrm{a}$ & $7,0 \mathrm{a}$ & $29,5 a$ & $50,0 \mathrm{a}$ & $54,7 \mathrm{a}$ \\
\hline 6 & $4,6 a$ & $6,5 \mathrm{a}$ & $6,8 \mathrm{a}$ & $32,0 \mathrm{a}$ & $49,0 \mathrm{a}$ & $50,8 \mathrm{a}$ \\
\hline 7 & $5,2 \mathrm{a}$ & $7,1 \mathrm{a}$ & $7,5 \mathrm{a}$ & $39,0 \mathrm{a}$ & $61,0 \mathrm{a}$ & $64,8 \mathrm{a}$ \\
\hline $\mathrm{CV}(\%)$ & 13,39 & 9,10 & 10,45 & 18,47 & 18,68 & 18,53 \\
\hline
\end{tabular}

${ }^{(1)}$ Médias seguidas da mesma letra não diferem entre si a $5 \%$ de probabilidade, pelo teste de Tukey.

Cavalcanti (1989), salienta-se, aqui, também em relação ao curuquerê.

Com relação à área foliar, aos $40 \mathrm{DAE}$ apenas o tratamento 7 diferiu significativamente dos demais (Tabela 3). Aos $60 \mathrm{DAE}$, o tratamento 7 diferiu significativamente dos tratamentos $1,3,4$ e 5; e aos 80 DAE, os tratamentos 1, 2, 3, 4 e 5 diferiram da testemunha.

Estes dados corroboram os já encontrados por Soares et al. (1997a, 1997b), que observaram haver um baixo desenvolvimento do algodoeiro quando destruídas as folhas da haste principal.

Quanto à área foliar, aos 40 DAE não houve diferenças significativas entre os tratamentos (Tabela 3 ). Aos 60 DAE, o tratamento 7 diferiu significativamente do tratamento 3 e aos 80 DAE, o tratamento 7 apresentou diferenças significativas em relação aos tratamentos $1,2,3$ e 5; e mostrou-se superior aos tratamentos 4 e 6 , porém, não diferindo dela significativamente. Embora aos 80 DAE não houvesse diferenças significativas entre os tratamentos $1,3,5$ e 6 , os tratamentos 1, 3 e 5 foram os que apresentaram os mesmos valores de área foliar.

De modo geral, nos tratamentos ímpares (1, 3 e 5), as lagartas de $A$. argillacea afetaram o crescimento e 0 desenvolvimento do algodoeiro pelo fato de terem destruído as folhas da haste principal as quais, além de serem as primeiras emitidas pela planta, são também responsáveis por mais de $80 \%$ da produção do algodoeiro (Soares et al., 1999).
Tabela 3. Resultados médios de área foliar $\left(\mathrm{cm}^{2}\right)$ em plantas de algodão cultivares CNPA 7H e CNPA Precoce 2 aos 40, 60 e 80 dias após a emergência (DAE), em 1997. Campina Grande, $\mathrm{PB}^{(1)}$.

\begin{tabular}{lccc}
\hline Tratamento & \multicolumn{3}{c}{ Área foliar } \\
\cline { 2 - 4 } & 40 DAE & 60 DAE & 80 DAE \\
\hline & & CNPA 7H & \\
1 & $419,26 \mathrm{~b}$ & $684,49 \mathrm{bc}$ & $843,80 \mathrm{~b}$ \\
2 & $456,47 \mathrm{~b}$ & $981,49 \mathrm{abc}$ & $1.060,01 \mathrm{~b}$ \\
3 & $377,87 \mathrm{~b}$ & $510,24 \mathrm{c}$ & $888,49 \mathrm{~b}$ \\
4 & $434,95 \mathrm{~b}$ & $795,44 \mathrm{bc}$ & $1.012,78 \mathrm{~b}$ \\
5 & $361,23 \mathrm{~b}$ & $772,11 \mathrm{bc}$ & $814,71 \mathrm{~b}$ \\
6 & $467,05 \mathrm{~b}$ & $1.113,94 \mathrm{ab}$ & $1.247,55 \mathrm{ab}$ \\
7 & $765,62 \mathrm{a}$ & $1.475,51 \mathrm{a}$ & $1.630,11 \mathrm{a}$ \\
\hline CV $(\%)$ & 24,80 & 24,85 & 18,08 \\
\hline & & CNPA Precoce & \\
1 & $621,01 \mathrm{a}$ & $892,07 \mathrm{ab}$ & $891,38 \mathrm{c}$ \\
2 & $596,00 \mathrm{a}$ & $966,16 \mathrm{ab}$ & $1.006,60 \mathrm{bc}$ \\
3 & $501,47 \mathrm{a}$ & $669,62 \mathrm{~b}$ & $956,62 \mathrm{c}$ \\
4 & $581,89 \mathrm{a}$ & $1.114,78 \mathrm{ab}$ & $1.622,61 \mathrm{ab}$ \\
5 & $623,68 \mathrm{a}$ & $1.161,26 \mathrm{ab}$ & $867,38 \mathrm{c}$ \\
6 & $676,77 \mathrm{a}$ & $896,18 \mathrm{ab}$ & $1.145,80 \mathrm{abc}$ \\
7 & $811,18 \mathrm{a}$ & $1.417,44 \mathrm{a}$ & $1.687,67 \mathrm{a}$ \\
\hline CV $(\%)$ & 38,49 & 23,89 & 23,89 \\
\hline
\end{tabular}

(1)Médias seguidas da mesma letra não diferem entre si a $5 \%$ de probabilidade, pelo teste de Tukey

\section{Conclusões}

1. A destruição das folhas da haste principal, por Alabama argillacea, reduz o crescimento do algodoeiro. 
2. Em relação à área foliar, o ataque de A. argillacea é mais severo após a floração.

3. O ataque de $A$. argillace a nas folhas dos ramos frutíferos é menos prejudicial ao algodoeiro do que nas folhas da haste principal.

\section{Referências}

ALVAREZ, R. J. A.; SANCHEZ, G. C. Alabama argillacea (Hüebner) ciclo de vida y consumo foliar. Revista Colombiana de Entomología, Bogotá, v. 8, p. 3438, 1982.

BELTRÃO, N. E. de M.; AZEVEDO, D. M. P. de. Defasagem entre as produtividades real e potencial do algodoeiro herbáceo: limitações morfológicas e ambientais. Campina Grande : Embrapa-CNPA, 1993. 108 p. (Documentos, 39).

BELTRÃO, N. E. de M.; CAVALCANTI, M. A. Crescimento e desenvolvimento do algodoeiro herbáceo, cultivar CNPA Precoce 1 no semi-árido paraibano e sua relação com o bicudo. Campina Grande : Embrapa-CNPA, 1989. 8 p. (Comunicado Técnico, 32).

BELTRÃO, N. E. de M.; AZEVEDO, D. M. P. de; VIEIRA, D. J.; NÓBREGA, L. B. da; QUEIROGA, V. P. de; SANTOS, J. W. dos; QUEIROZ, J. C. de; SOUZA, J. E. G. de. Observações morfológicas e agronômicas em algodoeiro arbóreo precoce: II. Frutograma de

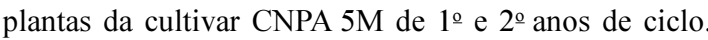
Campina Grande : Embrapa-CNPA, 1993. 11 p. (Comunicado Técnico, 36).

BELTRÃO, N. E. de M.; AZEVEDO, D. M. P. de; VIEIRA, D. J.; NÓBREGA, L. B.; QUEIROGA, V. de P.; SOUSA, J. E. G. de. Observações morfológicas e agronômicas em algodoeiro arbóreo precoce: I. Frutograma de plantas da cultivar CNPA 4M de 5o ano de ciclo. Campina Grande : Embrapa-CNPA, 1992. 5 p. (Pesquisa em Andamento, 14).

BLEICHER, E.; SILVA, A. L.; SANTOS, W. J. dos; GRAVENA, S.; NAKANO, O.; FERREIRA, L. Conheça os insetos da sua lavoura de algodão. Campina Grande : Embrapa-CNPA, 1983. 21 p. (Documentos, 3).

CAVALCANTI, R. D. O combate ao curuquerê do algodoeiro. Boletim Fitossanitário, São Paulo, n. 239, p. 1-2, dez. 1977.
CAVAlCANTI, R. D.; CAVAlCANTE, M. L. S. Duas sérias pragas do algodoeiro no Ceará. Fortaleza : EPACE, 1981. 8 p. (Comunicado Técnico, 6).

FALCON, L. A.; DAYL, R. Informe al algodonero de Nicaragua sobre control integrado de plagas del algodonero. Rome : FAO/UNDP, 1977. 285 p.

JÁCOME, A. G. Influência da remoção de folhas de algodoeiro (Gossypium hirsutum $L$.) no desenvolvimento vegetativo, rendimento e sua relação com o curuquerê (Alabama argillacea Hueb.). Areia : UFPB,1996. 55 p.

JÁCOME, A. G.; SOARES, J. J.; OLIVEIRA, R. H. de; CORDÃO SOBRINHO, F. P. Efeito da remoção de folhas no desenvolvimento vegetativo e na produção do algodoeiro. Pesquisa Agropecuária Brasileira, Brasília, v. 36, n. 5, p. 751-755, maio 2001 .

JOHNSEN, S. J. Larval development, consumption, and feeding behavior of the cotton leaf worm, Alabama argillacea (Hüebner). Southwestern Entomologist, College Station, v. 9, n. 1, p. 1-6, 1984.

LARA, F. M. Princípio de resistência de plantas a insetos. 2. ed. São Paulo : Ícone, 1991. 336 p.

MARCHINI, L. C. Avaliação de dano do curuquerê do algodão Alabama argillacea (Hübner, 1818) (Lepdoptera-Noctuidae) em condições similares e redução de sua população através de isca tóxicas. Piracicaba : ESALQ, 1976. 72 p. Dissertação de Mestrado.

NAKANO, O.; SILVEIRA NETO, S.; ZUCCHI, R. A. Entomologia econômica. São Paulo : Livroceres, 1981. $314 \mathrm{p}$.

NANKEN, L. N.; HEILMAN, M. D.; BROWN, R. C. Flowering intervals days to initial flower and seedling uniformity as factors for development of short season cotton cultivars. In: BELTWIDE COTTON PRODUCTION RESEARCH CONFERENCE, 1975, New Orleans. Proceedings... Memphis : National Cotton Council, 1975. p. 80-85.

OOSTERHUIS, D. M.; URWILER, M. J. Cotton mainstem leaves in relation to vegetative development and yield. Agronomy Journal, Madison, v. 80, n. 1, p. 65-67, 1988.

SILVA, A. L. da; PRADO, P. C. N. do; CUNHA, H. F. da. Manejo das principais pragas do algodoeiro em Goiás. Goiânia : EMGOPA, 1981. 19 p. (Circular Técnico, 2).

Pesq. agropec. bras., Brasília, v. 36, n. 8, p. 1005-1010, ago. 2001 
SOARES, J. J.; BUSOLI, A. C. Efeito dos reguladores de crescimento vegetal nas características agronômicas do algodoeiro e no controle de insetos. Pesquisa Agropecuária Brasileira, Brasília, v. 31, n. 1, p. 37-41, jan. 1996.

SOARES, J. J.; JÁCOME, A. G.; SOUSA, J. G. de; OLIVEIRA, R. H. de; WANDERLEY, D. S. Influência do desfolhamento simulado pelo ataque do curuquerê no desenvolvimento vegetativo e no rendimento do algodoeiro. Campina Grande : Embrapa-CNPA, 1997a. 6 p. (Comunicado Técnico, 61).
SOARES, J. J.; LARA, F. M.; SILVA, C. A. D. da; ALMEIDA, R. P. de.; WANDERLEY, D. S. Influência da posição do fruto na planta sobre a produção do algodoeiro. Pesquisa Agropecuária Brasileira, Brasília, v. 34, n. 4, p. 755-759, abr. 1999.

SOARES, J. J.; LARA, F. M.; SILVA, C. A. D. da; ALMEIDA, R. P. de; WANDERLEY, D. S. Observações morfológicas e agronômicas em algodoeiro herbáceo e suas relações com o manejo de pragas. Campina Grande : Embrapa-CNPA, 1997b. 6 p. (Pesquisa em Andamento, 39). 